\title{
Plastic deformation of beta-Ti-Mo alloys with isothermal omega phase
}

\author{
Koichi Tsuchiya ${ }^{*}{ }^{1}$, Satoshi Emura ${ }^{1}$, Xin $\mathrm{Ji}^{1}$, Ivan Gutierrez ${ }^{1}$, Toru Hara ${ }^{1}$ \\ 1: National Institute for Materials Science, Tsukuba, Japan
}

\begin{abstract}
Aging of metastable beta-Ti alloys leads to the precipitation of omega phase, resulting in a significant loss in ductility (omega brittleness). This presentation reports the results of 3D observations of isothermal omega phase by an orthogonal FIB/SEM system. It was revealed that the shape of omega phase particles varies with the particle size. By serial sectioning, it was possible to obtain a 3D image for the volume of several micrometer cube containing numerous omega particles. The volume fraction of omega phase was qualitatively determined. The value was much lower than the previous investigation by X-ray diffraction by Hickman in 1969 . The SEM and TEM revealed the formation of shear bands in the cold rolled samples, where the shearing of omega phase and transformation into beta phase while maintaining the depletion of Mo was found. After the HPT deformation, white etching layers are formed near the median plane of the disc, where the omega phase particles were absent and exhibit significantly lower hardness values. These findings may help to clarify the cause of brittleness in beta Ti alloys with isothermal omega phase.
\end{abstract}

\section{Introduction}

Isothermal aging of metastable beta-Ti alloys leads to the dense precipitation of so-called "isothermal omega phase", resulting in increase in hardness accompanied by a significant loss in ductility (omega brittleness). Due to their small size and high number density, the observation method of omega phase has been limited to transmission electron microscopy (TEM) and 3DAP. This report describes the results of 3D observations of isothermal omega phase particles by an orthogonal FIB/SEM system.

Cause of loss in deformability by omega phase was investigated by several researchers. Takemoto studied microstructures in tensile deformed Ti-14Mo alloy after isothermal aging at $623 \mathrm{~K}$ to obtain coarse $(80 \sim 100 \mathrm{~nm})$ omega particles[1]. Formation of deformation bands were observed, along which cracks seemed to preferentially propagate. The omega phase particles were not seen in the deformation bands and newly formed body centered triclinic phase was observed. More recently, Chen et al. studied the omega embrittlement in Ti-20Mo alloy. They have related to the extent of omega collapse to the change in the dislocation activity in beta phase from homogeneous slips to heterogeneous ones[2]. The present investigation also asses the mechanism of omega embrittlement by microstructural observation of the samples subjected to cold-rolling and also high-pressure torsion deformation.

\section{Experimental}

An ingot of Ti-12wt\%Mo were prepared by cold-crucible levitation melting (CCLM). It was then hot forged and rolled in to a form of a bar. An ingot of Ti-12wt $\%$ Mo were prepared by cold-crucible levitation melting (CCLM). It was then hot forged and rolled into a form of a bar. The plate samples were cut from the bar and homogenized at $1173 \mathrm{~K}$ for $3.6 \mathrm{ks}$. They were then aged at $673 \mathrm{~K}$ and $723 \mathrm{~K}$ for various time duration to obtain precipitation of isothermal omega phase. The plate sample was then subjected to cold rolling to the thickness reduction of $23 \%$. The morphology and distribution of omega phase was observed by cut and see observations using orthogonally arranged FIB-SEM (SMF-1000, SII NanoTechnology, Inc.) [3] were made with a FIB acceleration voltage of 30kV and a slicing pitch of $2 \mathrm{~nm}$ for $260 \mathrm{slices}$. Additional SEM observations were done on JEOL JSM-7001F. TEM observation were also carried out on JEOL JEM-2010F operated at $200 \mathrm{kV}$

\section{Results and Discussion}

Fig. 1(a) and (b) show the backscattered electron (BSE) images of samples aged at $673 \mathrm{~K}$ for $900 \mathrm{ks}((\mathrm{a}))$ and aged at $723 \mathrm{~K}$ for $180 \mathrm{ks}$. The particles with darker contrast is omega phase. The contrast in BSE images indicates the area are depleted with Mo which has higher atomic number than that of Ti. EDX analysis of omega particles revealed that the Mo content is about $7 \mathrm{wt} \%$. It can be also seen that the morphology of omega particles are different for different aging temperature.
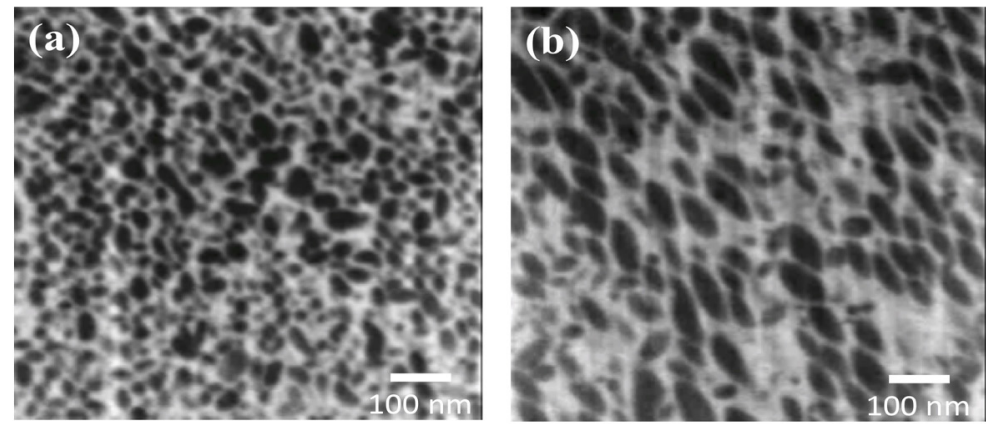

Fig. 1 Results of "Cut \& See" Observations of Ti-15Mo with w phase particles. (a) Aged at $673 \mathrm{~K}$ for 900 ks. (b) Aged at $723 \mathrm{~K}$ for 180 ks. w particles can be observed with high contrast and high resolution.
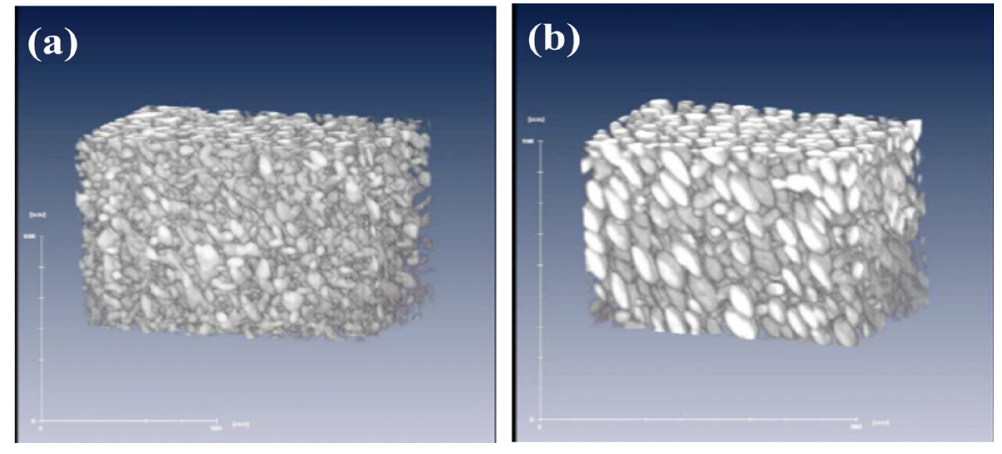

Fig. 2 3D Reconstructed Image of Ti - 15Mo. (a) aged at $673 \mathrm{~K}$ for $900 \mathrm{ks}$. (b) aged at $723 \mathrm{~K}$ for $180 \mathrm{ks}$.

The particles are more or less spherical in the sample aged at $673 \mathrm{~K}$ while they are ellipsoidal in the one aged at $723 \mathrm{~K}$. It can be further confirmed in the $3 \mathrm{D}$ reconstructed images shown in Fig.2 (a) and (b). Alignment of ellipsoidal particles in $723 \mathrm{~K}$ aged sample is also apparent. From these images, volume fraction of omega phase can be calculated and plotted in Fig. 3 together with the previous data by Hickman et al. The volume fraction of omega phase in the present study is very low compared to those by Hickman[4]. Hickman used single crystal X-ray diffraction and calculated volume fraction from peak intensity assuming all 4 variants of omega phase form equally. It may be possible that this leads to significant overestimation of the volume fraction. 


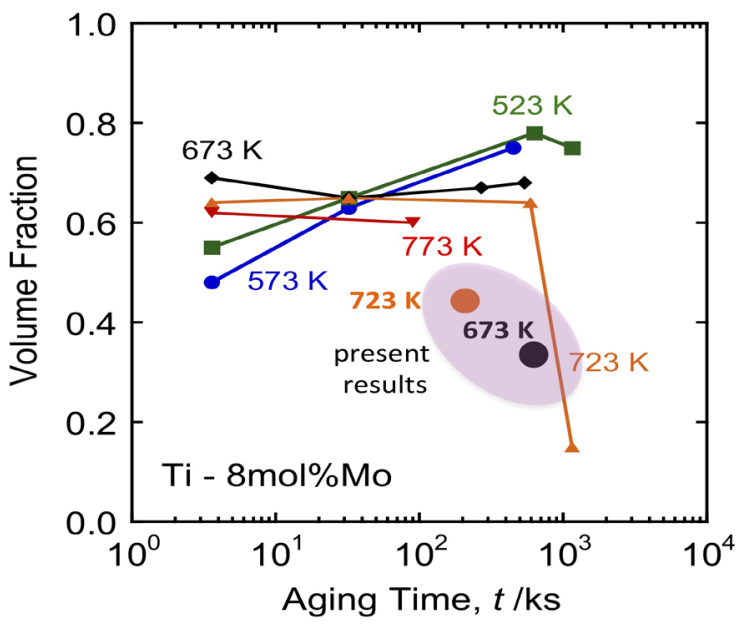

Fig. 3 Comparison of volume fraction of w phase. The results obtained by Hickman (XRD) are plotted with the present results.

Fig. 4 shows the BSE images of the sample aged at $723 \mathrm{~K}$ for $180 \mathrm{ks}$ and then cold-rolled. The sample was very brittle and broken in to pieces after rolling as seen in the inset. Fig.4 (a) is a low magnification BSE image of the cross section of the sample. Numerous shear bands can be seen with brighter contrast. Fig. 4(b) is a higher magnification BSE image of shear bands. It is seen that the matrix is filled with omega particles with darker contrast but omega particles are absent in the shear bands.
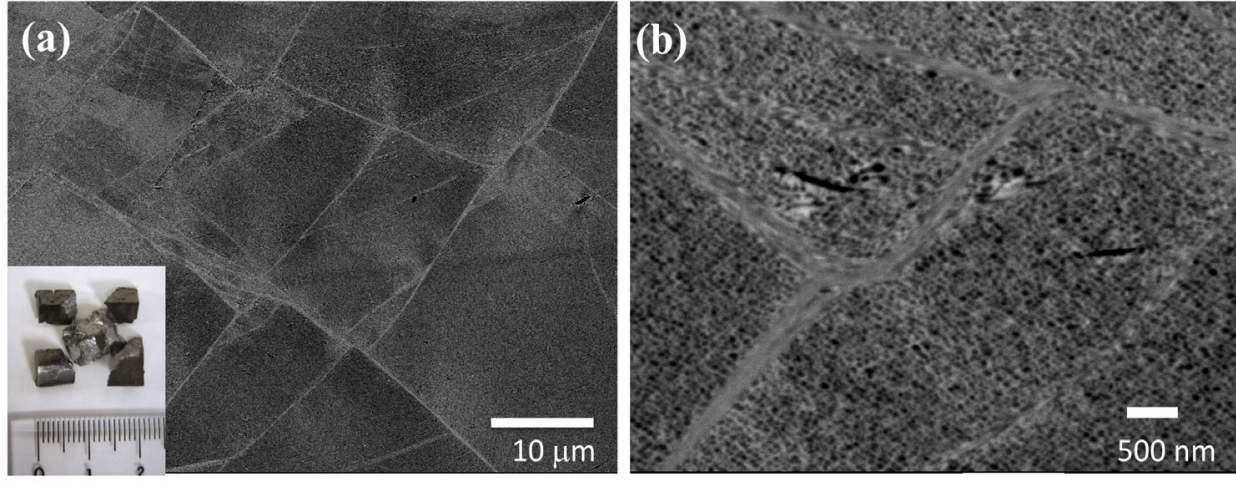

Fig.4 (a) SEM-BSE Image of Cold Rolled Ti-15Mo agied at $723 \mathrm{~K}$ for $180 \mathrm{ks}$ and cold rolled to $26 \%$.

In order to obtain more detailed information on the structure of the shear bands, TEM sample were obtained from the area containing shear bands by FIB. Fig. 5 (a)-(e) shows the results of TEM observations. Fig. 5(a) is a bright field (BF) image of area containing omega phase particles and two shear bands the widths of shear bands are about 30 $\mathrm{nm}$ (left) and $5 \mathrm{~nm}$ (right). Fig. 5(b) and (c) are selected area diffraction image obtained from an area in the matrix (A) and the one in the shear band (B). Fig. 5(b) shows the clear superlattice spots from the omega phase particles; while the omega spots cannot be seen in Fig.5(c). These observations strongly suggest that the omega phase particles transformed to beta phase by large shear deformation in the shear bands.

Lasalmonie et al. proposed the model of beta-to-omega transformation as the formation of three layers stacking faults [5]. Takemoto et al. [1] reported the reverse transformation of omega phase to beta phase tensile deformation. This transformation can occur by the glide of partial dislocations along successive three layers of (211) planes in the beta phase. The transformation from bcc phase to omega phase has been observed in shock-loaded deformation of Ta alloys [6].
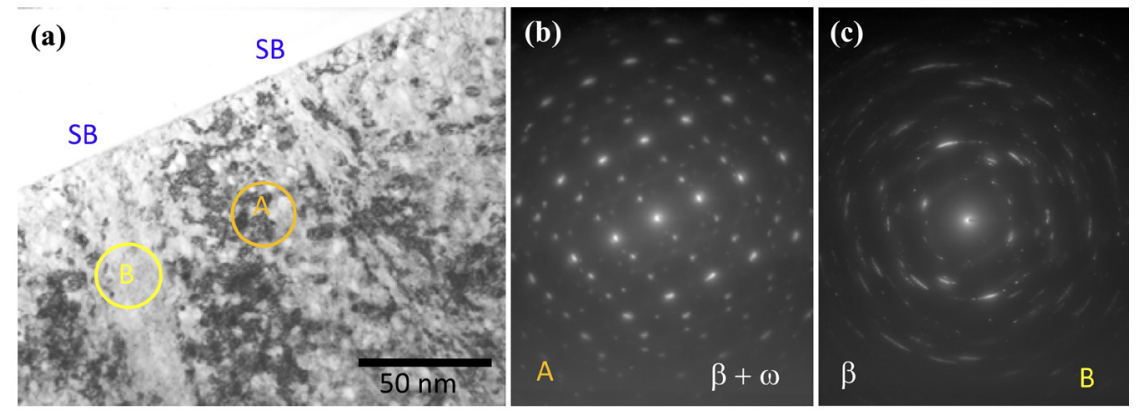

Fi. 5 (a) TEM observations of Ti- $15 \mathrm{Mo}$ after aged at $723 \mathrm{~K}$ for $180 \mathrm{ks}$ and cold rolled to $23 \%$. (a) bright field image. (b) SAD pattern from area A. (c) SAD pattnern from area B.

In our previous study, the microstructures and Vickers hardness of aged Ti-15Mo indicated similar omega-to-beta transformation in the shear bands and significant decrease in hardness in the shear bands [7]. Based on these results, In metastable beta Ti alloys containing isothermal omega phase particles, the deformation localizes into shear bands since the omega-to-beta transformation occurs by the motion of partial dislocation leading to significant softening. The localized deformation may cause significant stress concentration at the tip of shear bands reaches grain boundaries or other obstacles (alpha phase). This will cause nucleation of cracks. The present observations is in agreement of the mechanism of omega phase proposed by Takemoto et al. [1] 


\section{Conclusion}

Size and morphology of isothermal omega phase was observed by cut-and-see observation on orthogonal FIB-SEM. It was revealed that thw volume fraction of omega phase is much lower than that reported by Hickman. Microstructures of cold-rolled sample containing omega phase was investigated by SEM and TEM observations. The results indicate that the shear banding is the main mode of deformation which led to omega-to-beta transformation by partial dislocation and to significant softening. This is in agreement with the previously proposed model of the omega brittleness.

\section{Reference}

1. Takemoto, Y., M. HIda, E. Sukedai, A. Sakakibara, Jounal Japan Inst. Met. 53, 1004-1012 (1989).

2. Chen, W. et al., Acta Mater. 170, 187-204 (2019)

3. Hara, T. et al., J. Alloys Compd. 577, (2013).

4. Hickman, B. S., Met Soc AIME-Trans 245, 1329-1336 (1969).

5. Lasalmonie, A. \& Chaix, C., Philos. Mag. A Phys. Condens. Matter, Struct. Defects Mech. Prop. 44, 973 (1981)

6. Hsiung, L. M. \& Lassila, D. H., Acta Mater. 48, 4851-4865 (2000).

7. Farjami, S., Tsuchiya, K., Todaka, Y. \& Umemoto, M. in Processing and Fabrication of Advanced Materials - XVIII (eds. Niinomi, M., Morinaga, M., Nakai, M., Bhatnagar, N. \& Srivatsan, T. S.) 1053-1060 (2009). 